Journal for ImmunoTherapy of Cancer

\title{
Targeting cancer testis antigens in synovial sarcoma
}

\author{
Geoffrey Mitchell, ${ }^{1}$ Seth M Pollack (D) , ${ }^{2,3,4}$ Michael J Wagner (1) ${ }^{2,3}$
}

To cite: Mitchell G, Pollack SM, Wagner MJ. Targeting cancer testis antigens in synovial sarcoma. Journal for ImmunoTherapy of Cancer 2021;9:e002072. doi:10.1136/ jitc-2020-002072

Accepted 04 May 2021
Check for updates

(c) Author(s) (or their employer(s)) 2021. Re-use permitted under CC BY-NC. No commercial re-use. See rights and permissions. Published by BMJ.

${ }^{1}$ University of Washington, Spokane, Washington, USA ${ }^{2}$ Clinical Research Division, Fred Hutchinson Cancer Research Center, Seattle, Washington, USA ${ }^{3}$ Oncology, University of Washington, Seattle, Washington, USA

${ }^{4}$ Lurie Cancer Center, Northwestern University, Chicago, Illinois, USA

Correspondence to Dr Michael J Wagner; wagnermj@uw.edu

\section{ABSTRACT}

Synovial sarcoma (SS) is a rare cancer that disproportionately affects children and young adults. Cancer testis antigens (CTAs) are proteins that are expressed early in embryonic development, but generally not expressed in normal tissue. They are aberrantly expressed in many different cancer types and are an attractive therapeutic target for immunotherapies. CTAs are expressed at high levels in SS. This high level of CTA expression makes SS an ideal cancer for treatment strategies aimed at harnessing the immune system to recognize aberrant CTA expression and fight against the cancer. Pivotal clinical trials are now underway, with the potential to dramatically alter the landscape of SS management and treatment from current standards of care. In this review, we describe the rationale for targeting CTAs in SS with a focus on NY-ESO-1 and MAGE-A4, the current state of vaccine and T-cell receptor-based therapies, and consider emerging opportunities for future development.

\section{INTRODUCTION}

\section{Synovial sarcoma}

Synovial sarcoma (SS) is a rare cancer that comprises between $5 \%-10 \%$ of all soft tissue sarcomas that disproportionately affects children and young adults, with the peak incidence between 15-29 years old, though it can occur at any age. ${ }^{1-3}$ Although it was originally thought to derive from synovium when first described over 100 years ago, ${ }^{4}$ SS can develop from any serosal surface including the pleura or sac around the kidney. One hypothesis regarding its cell of origin is that it derives from Myogenic factor 5 expressing multipotent mesenchymal progenitors such as periosteal cells and pre-osteoblasts influenced by paracrine secretion of osteoprotegerin by bone, suggesting why SS often form immediately adjacent to bone. ${ }^{5}$ Morphologically, SS can present in two distinct histological subtypes: monophasic, which is composed uniformly of spindle cells or biphasic, which contains areas with epithelioid differentiation.

Patients with localized disease are treated with curative intent with surgical resection and radiation therapy. Anthracycline-based chemotherapy is often used in the curative setting and is the primary treatment used in the metastatic setting. Regardless of presentation, 5-year and 10-year overall survival (OS) is $61 \%$ and $42 \%$, respectively. ${ }^{1}$ While patients with localized disease can be cured, patients with disease that has recurred or who have metastatic disease have far worse outcomes. The use of ifosfamide, ${ }^{6}$ pazopanib, $^{7}$ and trabectedin $^{8}$ have improved outcomes for anthracycline refractory patients but profession-free survival with any of these drugs can be measured on the order of months. In spite of great progress with multiple new drugs developed over the past decade, median OS for patients receiving systemic therapy is around 2 years. ${ }^{9}$

$\mathrm{SS}$ is characterized by one of several $\mathrm{t}(\mathrm{X} ; 18)$ translocations that is the pathognomonic driver for the tumor and leads to expression of an abnormal SS18-SSX (previously SYT-SSX) fusion protein. ${ }^{110}$ Recent insights into the mechanism of the SS18-SSX fusions have led to a better understanding of the biology of SS and highlighted the role of chromatin remodeling in SS pathogenesis. SS18 is a normal component of barrier-toautointegration factor (BAF)-type Switch/ Sucrose Non-Fermentable (SWI/SNF) complexes. ${ }^{11}$ SS18-SSX fusions interact with transducin-like enhancer protein 1 and activating transcription factor 2 (ATF2) leading to repression of ATF2 target genes. ${ }^{12}$ The fusion protein ejects the SMARCB1 (BAF47, INI1) subunit from the BAF complex and leads to altered transcription of genes including HOXC10, BCL2, PAX7, and SOX2, ultimately leading to cancer cell survival. ${ }^{13-15}$ As described below, SS highly expresses cancer testis antigens (CTAs). Although the mechanisms by which CTAs are expressed in cancer are not clear, epigenetic dysregulation mediated by SS18-SSX fusion proteins may play a key role.

\section{CTAs}

It has long been recognized that tumors can elicit immune responses. Early efforts to identify tumor associated antigens in a murine model revealed four antigens that 
were recognized by syngeneic cytotoxic T-lymphocytes (CTLs) and whose expression was required to maintain the host immune response and prevent tumor progression. ${ }^{16}$ Translation of this finding led to the identification of the first human T-cell antigen in melanoma, melanoma antigen-1 (MAGE-1, renamed MAGE-A1). ${ }^{17}$ Additional immunogenic antigens, notably including NY-ESO-1, were identified by screening complementary DNA (cDNA) expression libraries with autologous CTLs (serological analysis of recombinant cDNA expression libraries or SEREX). ${ }^{18}$ After multiple gene families were identified with expression seemingly restricted to cancer and testis, the group of gene families collectively were termed CTAs. ${ }^{19}$ To date, over 70 CTA gene families have been identified. ${ }^{20}$

Work to elucidate the function of CTAs implicates them in sperm and oocyte biology. Synaptonemal complex protein 1 , a CTA implicated in ovarian cancer, ${ }^{21}$ is a normal component of the synaptonemal complex required for meiosis. ${ }^{22}$ NY-ESO-1, a CTA expressed in several cancer types and one of the most relevant for cancer therapeutics targeting CTAs, is expressed in testis and ovary during embryonic development with expression levels peaking in the second trimester. ${ }^{23}$ MAGE family genes play a role in cell cycle control and neurogenesis. ${ }^{24}$ The mechanisms for aberrant expression in cancers remains uncertain, but may be at least in part related to aberrant demethylation at the promotor of CTA genes. ${ }^{25}$ High expression of CTAs is mostly limited to developing embryos, placental trophoblasts, and to immunoprotected tissues such as testis in adults with very low but detectable expression in some other tissues. ${ }^{26}$ After their discovery, it became clear that CTAs are expressed to varying degrees in many different cancer types. ${ }^{27}$ These include melanoma, ${ }^{28}$ breast cancer, ${ }^{29}$ ovarian cancer, ${ }^{30}$ bladder cancer, ${ }^{31}$ lung cancer, ${ }^{32} 33$ myeloma, ${ }^{34}$ and hepatocellular carcinoma. ${ }^{35}$ Importantly, the observation of spontaneous humoral immune responses to CTAs suggested the possibility that they are an attractive target for cancer immunotherapy with vaccines and adoptive T-cell strategies. The first vaccine trials demonstrated that CD8 T-cell specific responses could be elicited with vaccination with NY-ESO-1 peptide, with hints of clinical benefit. ${ }^{36}$

\section{CTAs in SS}

In contrast to most other cancer types, CTA expression in SS is exceptionally high. Additionally, CTA expression in SS is homogenous. Few cancer types, with the exception of myxoid/round cell liposarcoma, ${ }^{37}$ demonstrate this unique pattern of CTA expression. Table 1 highlights specific studies that have quantified the expression of CTAs that have been found in SS. Of note, although RT-PCR was used to identify CTA expression in the past, transcript expression does not always correlate with protein expression and immunohistochemistry (IHC) is now the gold standard. ${ }^{28}$ The expression of CTAs in SS is a dynamic process with some patients potentially showing expression later in the disease course compared with others. With this in mind, care must be taken when determining the eligibility of patients for CTA-targeted treatments. As the CTAs that have been best characterized in SS, we focus mostly on NY-ESO-1 and MAGE-A4 in this review.

NY-ESO-1 is perhaps the best characterized CTA in SS. While the normal function of the protein is unknown, NY-ESO-1 has been shown to interact with MAGE-C1 and may be important in tumor cell proliferation and tumor survival by inhibition of p53. ${ }^{38-40}$ There have been multiple studies that have confirmed the presence and incidence of NY-ESO-1 in SS with ranges from $49 \%$ to $82 \%$ with IHC, as well as historically with PCR and microarray. ${ }^{41-47}$ Estimates on the lower end of the spectrum may be underestimating the true incidence of NY-ESO-1 expression by assessing limited amounts of tumor in a tissue microarray. ${ }^{44}$ Although NY-ESO-1 expression tends to be heterogenous on most tumor types and can be heterogenous on SS, it is often homogenously expressed in SS. This homogenous expression makes SS an exceptional candidate for NY-ESO-1 targeted therapies. ${ }^{43}$

The MAGE-I family of CTAs consists of MAGE-A, MAGE-B, and MAGE-C. Each of these then have subfamilies and are numbered accordingly. The MAGE-A family directly inhibits the function of p53 by binding to the DNA binding portion of $\mathrm{p} 53 .^{48}$ It also leads to increased levels of MDM4, a p53 inhibitor. ${ }^{49}$ MAGE-A4 is a CTA that has been shown to be expressed in $53 \%-82 \%$ of SS by IHC. ${ }^{41}{ }^{42}$ MAGE-A4 is the most relevant of the MAGE family genes for SS. NY-ESO-1 and MAGE-A4 are often expressed in the same tumor, but the two can be expressed independent from one another as well. ${ }^{42}$

Preferentially Expressed Antigen In Melanoma (PRAME) expression has been detected in $86 \%-100 \%$ of SS, though IHC assays for PRAME are not as reliable as those for NY-ESO-1 or MAGE-A4. ${ }^{41} 4750-52$ PRAME aids in tumor survival by inhibiting apoptosis, proliferation arrest, and retinoic acid induced differentiation in two ways. ${ }^{53}$ PRAME can bind to the retinoic acid receptor directly or can complex with the polycomb group protein EZH2 to suppress retinoic acid receptor signaling. ${ }^{53}$ Importantly, T-cell responses against PRAME have been characterized in other tumor types. ${ }^{54} \mathrm{Up}$ to this point there are no clinical studies of $\mathrm{T}$ cells being used to target PRAME in SS. There are currently two separate ongoing trials (NCT04262466 and NCT03686124) using T-cell receptors (TCRs) to target PRAME positive solid cancers.

The related SSX genes SSX1 and SSX2, the fusion partners defining the SS18-SSX SS translocation, have long been considered CTAs due to their expression being solely in the male testis and location on the X chromosome. ${ }^{55}$ The presence of SSX1 and SSX2 in the pathognomonic fusion protein in SS makes SSX a potential target for both vaccines and modified T cells. ${ }^{56}$ 
Table 1 Cancer testis antigen (CTA) expression in synovial sarcoma (SS)

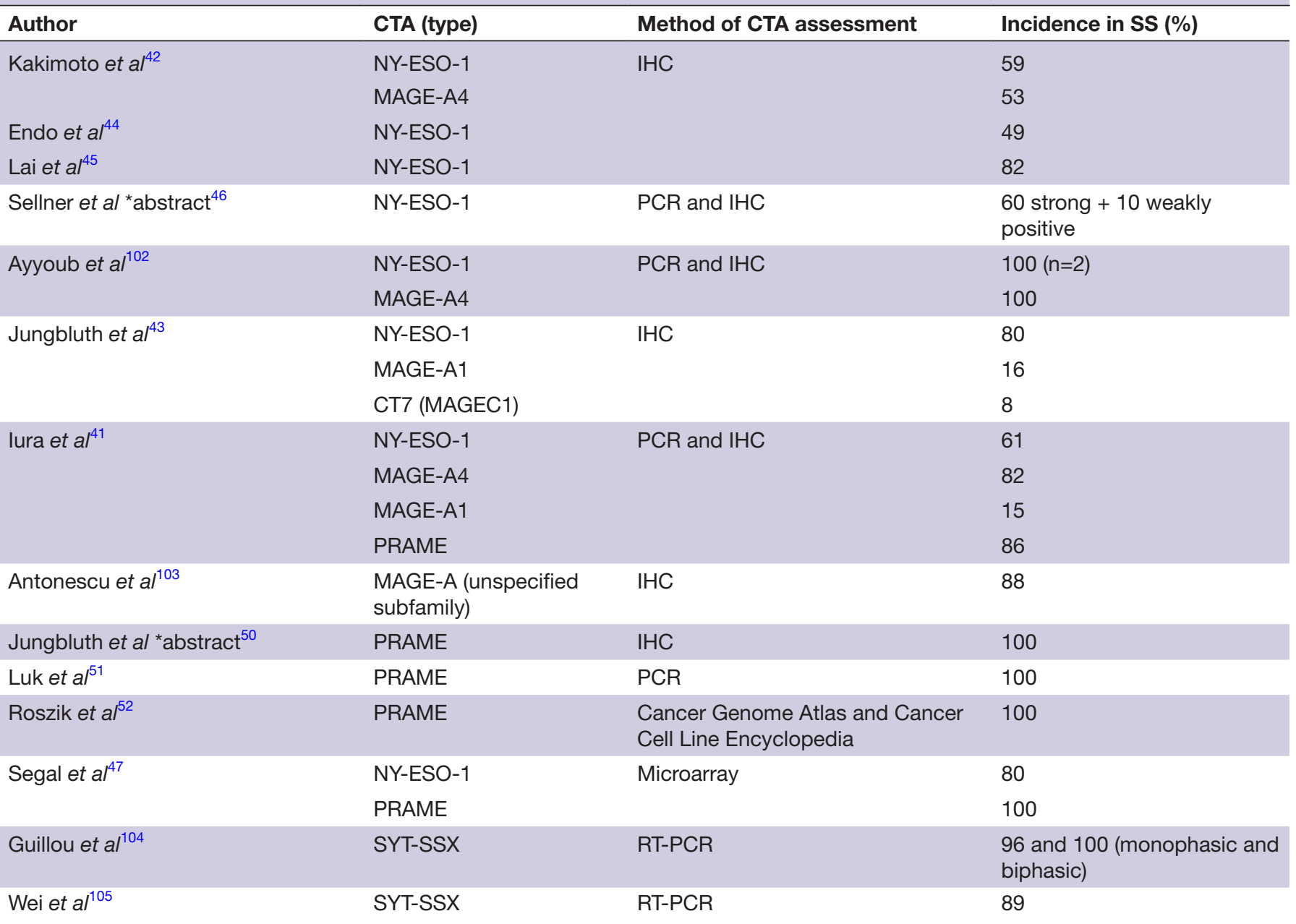

IHC, immunohistochemistry; PRAME, Preferentially Expressed Antigen In Melanoma.

\section{TARGETING CTAS IN SS}

Interventional trials targeting CTAs in SS are summarized in table 2.

\section{CTA-targeted vaccines}

LV305 was a vaccine that targets dendritic cells and induces the expression of NY-ESO-1 to then stimulate T-cell

Table 2 Interventional studies of CTA-targeted therapeutics in synovial sarcoma, grouped by therapy type

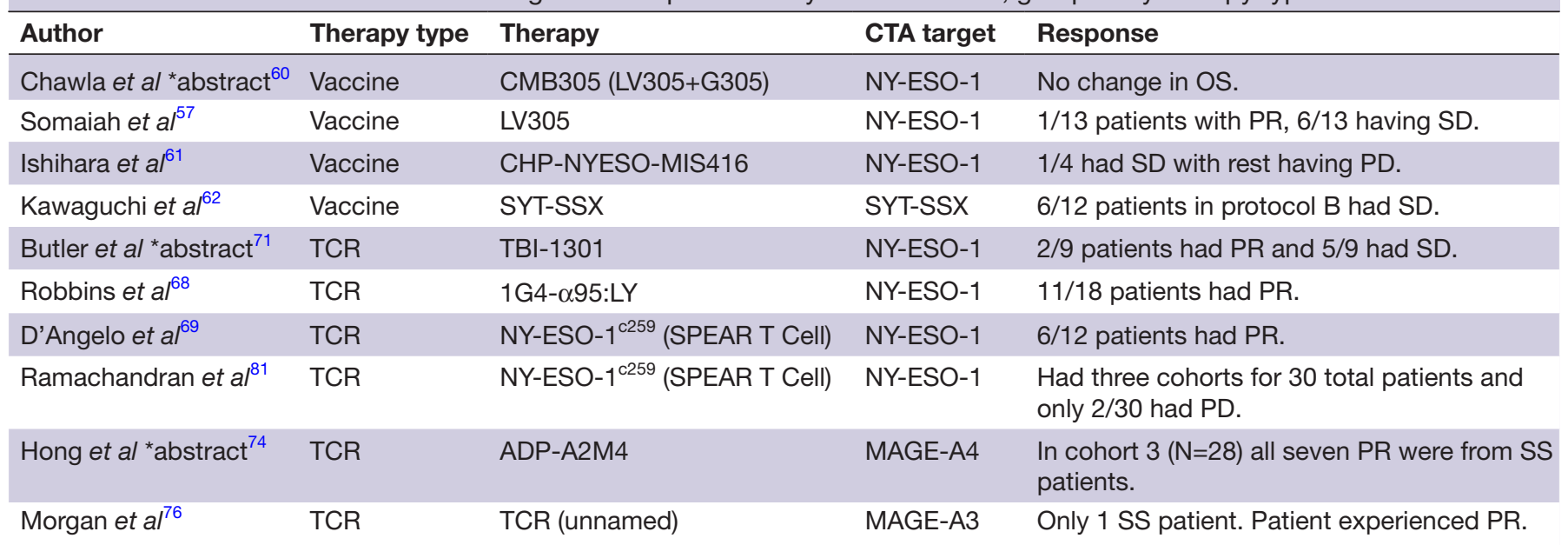

CTA, cancer testis antigen; OS, overall survival; PD, progressive disease; PR, positive response; SD, stable disease; SS, synovial sarcoma; TCR, T-cell receptor. 
mediated immune response.$^{57}$ Initial results of LV305 in a phase 1 trial found that $62 \%$ of patients had stable disease as their best response with one patient (4\%) having a complete response. ${ }^{57}$ Patients with increased clonal expansion of anti-NY-ESO-1 T cells induced by LV305 were also shown to have a significantly longer OS. Efforts to improve the efficacy of LV305 were undertaken with a modified version of LV305, called CMB305. CMB305 includes LV305 boosted with an NY-ESO-1 recombinant protein plus glucopyranosyl lipid A, a TLR-4 agonist, in a stable emulsion (GLA-SE) ${ }^{58}$ In an initial Phase 1 trial, CMB305 was safe and elicited antibody responses against NY-ESO-1 in $62.9 \%$ of patients and T-cell responses in $47.4 \% ; 22.8 \%$ of patients had both. ${ }^{59} \mathrm{~A}$ randomized Phase 2 study with the programmed death-ligand 1(PD-L1) inhibitor atezolizumab with or without CMB305 showed no statistically significant differences between the groups in OS and progression free survival (PFS), and no further study is planned with this vaccine regimen. ${ }^{60}$

CHP-NYESO-MIS416 is a vaccine which is a polysaccharide-ligated to NY-ESO-1 used to stimulate MHC I and 2 with a T cell stimulator (MIS416), with NOD2 and TLR9 added as stimulants. ${ }^{61}$ In a study of this vaccine across multiple cancer types, only one out of a total of four patients with SS had stable disease (SD) with the rest having progressive disease (PD) as best response. While this was a small cohort all four patients with SS developed a specific immune response as assessed by the presence of NY-ESO-1 specific antibodies. An additional mouse model using the CHP-NYESO-MIS426 vaccine in conjunction with an anti- programmed cell death protein 1 (PD1) inhibitor showed a significant decrease in tumor volume, suggesting that co-administration of this vaccine and PD1 inhibitors may be an effective treatment strategy. ${ }^{61}$

Vaccine therapies have also had their own difficulties. The induction of CD8+ lymphocytescan take up to several months to occur and limits the population of patients who can use this method of therapy. ${ }^{57}$ Due to the aggressive nature of SS, the utility of a vaccine-based therapy in patients with rapidly progressing disease is limited. Efforts to increase the efficacy of vaccines have used checkpoint inhibitors, synthetic TLR4, and Freund's adjuvant to increase and boost the cytotoxicity of the stimulated T cells. ${ }^{6062}$ In addition to stimulating humoral immunity, vaccination with NY-ESO-1 vaccine in metastatic melanoma also triggered an increase in regulatory T-cells that recognize NY-ESO-1 suggesting a mechanism of resistance. ${ }^{63}$

In an effort to increase the efficacy of vaccines, treatment with Poly-ICLC, which is a TLR3 and MDA5 agonist, along with Montanide, an oil-based vaccine adjuvant, and a NY-ESO-1 vaccine increased immunogenicity of the vaccine in patients with melanoma. ${ }^{64}$ This combination induced $\mathrm{CD} 4+$ and $\mathrm{CD} 8+\mathrm{T}$ cells that were able to produce interferon (IFN) $\alpha$, IFN $\gamma$, and interleukin (IL)-2. ${ }^{64}$ While this was not studied in patients with SS, the high level of NY-ESO-1 in SS makes this an ideal population for this approach.

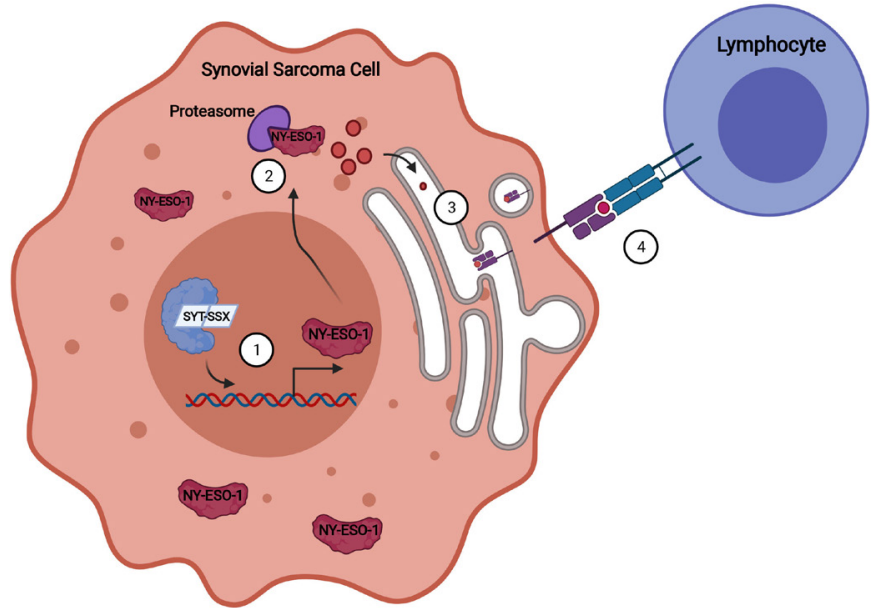

Figure 1 Aberrant expression of NY-ESO-1 and presentation on MHC. The presence of the SYT-SSX fusion protein leads to abnormal epigenetic regulation in the synovial sarcoma cell, causing aberrant NY-ESO-1 expression (1). NY-ESO-1 is degraded in the cytoplasm (2) and transferred into the endoplasmic reticulum for processing where NY-ESO-1 peptides are bound to MHC molecules (3). The peptide-MHC complex is presented on the surface of the cell and recognized by the TCR of the transduced lymphocytes (4).

\section{T-cell therapies}

Autologous T-cell therapies developed for SS are generated from T-cells isolated from a patient's peripheral blood and either expanded ex vivo ${ }^{65}$ or modified in vitro $^{66}$ to recognize a specific CTA, and then infused back into the patient after conditioning chemotherapy. T-cell therapies have had great success in the clinic for multiple cancer types, particularly chimeric antigen receptor (CAR) T cells for hematological malignancies. Most T-cell therapies for SS studied to date are autologous $\mathrm{T}$ cell products with human leukocyte antigen (HLA) restricted TCRs. The general mechanism of TCRs in SS is shown in figure 1. In 2008, Robbins et al published seminal work in which they isolated the 1G4 TCR and demonstrated that CDR $3 \alpha$ and CDR2 $\beta$ amino acid substitutions enhance the antigen specific activity of the modified T-cells. ${ }^{67}$ These cells, named 1G4- $\alpha 95: \mathrm{LY}$, are made with a retroviral vector to recognize the peptide SLLMWITQC, which is residue 157 to 165 of the NY-ESO-1 protein. They were assessed in the clinic given after lymphodepletion as cell infusion alone and combined with IL-2 for T-cell stimulation. Sixty-one per cent (11) of patients had a partial response, one of which was a complete response. ${ }^{66} 68$ Interestingly, prevalence of NY-ESO-1 T-cells 1 month after treatment did not correlate with the observed clinical responses. ${ }^{68}$

NY-ESO- $1^{\text {c259 }}$ is another modified TCR that has shown promise in two recent trials. The NY-ESO- ${ }^{\text {c259 }} \mathrm{T}$ cells are generated from CD15 depleted CD4+ and CD8+ cells that, like other TCR products, are then transduced with a lentiviral vector to express a TCR recognizing the SLLMWITQC epitope of NY-ESO-1. Results of an initial trial in patients with SS demonstrated that half of the patients 
responded with one patient having a complete response. ${ }^{69}$ The median OS was 120 weeks. Importantly this study documented that the maximal antitumor response in four patients occurred 3 months after starting therapy. This showed that there is a continued immune mediated response against the tumor mediated by in vivo expansion and persistence of the NY-ESO- $1^{\text {c259 }}$ cells after infusion. ${ }^{69}$ Patients receiving NY-ESO-1 modified T cells have also shown evidence of neurotoxicity, with two patients developing Guillain-Barre syndrome related to their treatment, but overall the toxicities seen with NY-ESO-1 TCRs are on par with other patients receiving lymphodepleting chemotherapy. ${ }^{70}$ Other TCR's, such as TBI-1301, are in clinical development and may have evidence of clinic benefit based on publicly presented data. ${ }^{72}$ In addition to a reduction in tumor size, infusion of both of these cell products led to cytokine release syndrome, as is often seen with effective cell-based therapeutics. ${ }^{71} 72$ Larger scale clinical trials with these other cell products are eagerly awaited.

T-cells targeting MAGE-A4 have also made it to the clinic in larger scale clinical trials for patients with SS. There are currently three clinical trials involving $\mathrm{T}$ cells targeting MAGE-A4 involving patients with SS that are ongoing (NCT03132922, NCT04044768, NCT04044859). These studies use ADP-A2M4 which is a T-cell targeting MAGE-A4 peptide GVYDGREHTV. Promising in vitro and in vivo efficacy was seen in a mouse model. ${ }^{73}$ In the clinic, an initial report demonstrated clinical activity; $25 \%$ (7) of the patients had a PR. All seven of the patients who had a response had SS. ${ }^{74}$ Longer-term follow-up of patients with SS specifically demonstrated a response rate of $44 \%$, with durable responses lasting at least 6 months. ${ }^{75}$ Preliminary translational analysis showed that MAGE-A4 expression levels and the total cell infusion dose correlated with response. ${ }^{75}$ Also within the MAGE family, a TCR targeting MAGE-A3 had clinical efficacy in one patient with SS enrolled on a larger multi-histology trial; but neurotoxicity thought to be secondary to cross reactivity with MAGE-A12 may limit further clinical development with this CTA as a target. ${ }^{76}$

TCR editing using the CRISPR-Cas9 system may allow for optimization of the autologous T-cells to maximize the likelihood of a sustained antitumor response after cell infusion. The use of CRISPR engineered $T$ cells against sarcoma has so far been limited, with the results of one phase I trial showing tumor evasion. ${ }^{77}$ These CRISPR engineered T cells, modified to express an NY-ESO-1 TCR and removal of the gene encoding PD-1, demonstrated long persistence for up to 9 months with no significant toxicity. There was also only one patient with sarcoma in this phase 1 trial, but this patient experienced the longest duration of SD observed. ${ }^{77}$ Additional advances such as the ability to reduce off-target editing by Cas 9 and increase the specificity of gene targeting may ultimately lead to improvements in TCR development and production. ${ }^{78}$

A major challenge of TCR therapies is the restriction of these modified T-cells to target patients who are HLA
$\mathrm{A} * 02: 01$ positive. HLA A*02:01 has the highest incidence within the USA in Caucasian populations with lower expression levels observed in Asian and African-American populations. ${ }^{79}$ This restriction limits the population that could possibly receive this treatment and leaves many other patients with little help beyond conventional methods, leaving a large unmet need for patients with other HLA haplotypes. They also require significant time for cell processing which can take on average 1-2 months to modify and grow the $\mathrm{T}$ cells before they are ready to be used.

\section{Role of conditioning regimen in T-cell therapies}

Although the TCR is the main active anti-sarcoma entity in adoptive T-cell therapy, the studies of NY-ESO-1 targeted T-cells highlighted the important role of the conditioning regimen for T-cell activity. Lymphodepleting immunomodulating drugs, such as fludarabine and cyclophosphamide, are used to deplete pre-existing lymphocytes that can compete with the transferred T cells. ${ }^{80}$ This lymphodepletion eliminates CD4+CD25+ T-regulatory lymphocytes and competition for endogenous cytokines and gives the transferred $\mathrm{T}$ cells an advantage to survive and populate in the host bone marrow. This process of depletion is done prior to treatment and increases the efficacy and numbers of lymphocytes in the tumor microenvironment (TME) after cell infusion. ${ }^{80}$ Fludarabine specifically has been shown to have the greatest effect on the $\mathrm{T}$ cell cytokines IL-7 and IL-15. ${ }^{6980} 81$ An expansion study of the NY-ESO- ${ }^{\text {c259 }}$ cells sought to assess the activity of these cells in patients with lower expression level of NY-ESO-1 or with alternative pre-treatment lymphodepleting chemotherapy regimens. ${ }^{81}$ This resulted in fewer responses when comparing the expansion cohorts to the previous trial with higher intensity conditioning including fludarabine; with the initial trial having a response rate of $50 \%$ and the expansion cohorts having a combined response rate of $30 \%$. These expansion cohorts demonstrated that more aggressive lymphodepletion may increase the likelihood for the success of TCR therapies and highlight the importance of fludarabine, ${ }^{81}$ which would otherwise not be used to treat SS. The intensive conditioning that patients must go through before receiving T-cells limits the patient population to those with good performance status. Older patients or those who have received multiple highly myelosuppressive regimens may have less bone marrow reserve and be less likely to tolerate the more effective higher intensity regimens, with fatal aplastic anemia reported in early studies. ${ }^{745}$

\section{SS MICROENVIRONMENT}

CTA-targeted therapies such as vaccines and cell-based therapies are reliant on a favorable TME to exert their intended antitumor effects. The microenvironment consists of factors both intrinsic to the cancer cell such as secretion of immune modulating cytokines and expression of immune checkpoint inhibitors, and extrinsic such 
as lymphocytes and macrophages, all of which interact with each other and create a microenvironment that can be immunogenic or 'cold'. Immunologically 'cold' tumors are felt to be less suited for checkpoint inhibitors. ${ }^{82}$ Tumors with a high tumor mutation burden (TMB) tend to be more immunogenic. SS is an immunologically 'cold' tumor with a low $\mathrm{TMB}^{83}$ - a problem that must be overcome to maximize the benefit of CTAtargeted therapies.

SS has a low level of tumor infiltrating T cells (TIL) ${ }^{84-87}$ While in some inflammatory tumors like melanoma TIL are a marker for better prognosis, ${ }^{85} 86$ there is evidence suggesting that higher levels of CD8+ lymphocytes in the TME of SS might lead to worse metastasis free survival. ${ }^{88}$ Wedekind, et al in particular found that recurrent or metastatic tumors were less likely to have high TIL infiltration, suggesting that the tumor may acquire changes allowing it to evade the immune system. ${ }^{87}$ One potential clinical consequence of this finding is that immune modulating treatment earlier in the patient's course of disease might be of greater benefit than waiting for recurrence. ${ }^{87} \mathrm{PD}-\mathrm{L} 1$, PD-1, and CD8+ T cells are enriched at the perimeter of SS tumor, but relatively low inside the tumor. ${ }^{89}$ NY-ESO-1 expression levels are not correlated to CD8+ lymphocyte density in the tumor, indicating that the presence of CTAs in the tumor cells are not enough to stimulate endogenous cytotoxic T-cells into the tumor. ${ }^{89}$

HLA expression in the tumor is crucial for the functioning and efficacy of adoptive $\mathrm{T}$ cells to recognize their antigen target and attack the tumor. SS generally has low HLA expression. ${ }^{51} 848687$ Biphasic SS has higher HLA I expression in the epithelioid components compared with monophasic SS; 5 out of 10 patients had focal high expression in biphasic tumors compared with 1 out of 16 patients with monophasic SS. ${ }^{51}$ The overall deficiency of HLA I in SS has led to the idea that this might be a main mechanism by which SS is able to evade the immune system from either its own in vivo stimulation or via ex vivo therapeutics. ${ }^{84}{ }^{87}$ Giving IFN $\gamma$ in patients with SS has been shown to increase the levels of HLA I expression in the tumor cells along with increasing the density of TIL. ${ }^{90}$ A phase 0 trial in patients with SS and myxoid/round cell liposarcoma (MRCL) designed to test whether IFN $\gamma$ could increase MHC expression and $\mathrm{T}$ cell infiltration found that it $\operatorname{did} .^{91}$ Based on these results IFN $\gamma$ was combined with NY-ESO-1 specific T cell therapy in a clinical trial but had a fatal complication. ${ }^{92}$ Subsequently, a multicenter trial combining IFN $\gamma$ with PD-1 inhibition has been completed and data analysis is ongoing (NCT03063632).

There is some evidence that an immune reaction to CTAs can be instigated following treatment with checkpoint inhibitors. ${ }^{93}$ The presence and density of PD-1 and PD-L1 are correlated with a poorer prognosis for SS. ${ }^{89}$ While PD-L1 has been shown to be expressed in some SS tumors, the expression levels are typically low. ${ }^{88}$ PD-L1 is more highly expressed in metastatic or recurrent SS and its expression correlates with a shorter PFS. ${ }^{87} 89$ In a small clinical trial of ipilimumab in patients with advanced SS, there were hints of an immune response against MAGE-A3 and MAGE-A4 in response to checkpoint inhibition. This patient had detectable antibodies against these antigens prior to treatment, suggesting that immune checkpoint blockade may heighten pre-existing immune reactions against CTAs. Interestingly, this patient also had a seroconversion with newly detectable antibodies against CSAG2, another CTA, after treatment with ipilimumab. One patient (of 10 total with SS) responded to pembrolizumab on the SARC28 study. ${ }^{94}$ The low number of patients with SS in these studies demonstrating clear clinical benefit suggests that immune checkpoint inhibition alone is not sufficient to elicit clinically meaningful immune responses against CTAs.

SS is angiogenic with a predilection for formation of abnormal tumor vasculature. ${ }^{95}$ Dysregulated angiogenesis impedes immune cell trafficking, and co-administration of anti-angiogenic drugs with immune checkpoint inhibitors has been promising in preclinical models and in the clinic in other cancer types. ${ }^{96}$ The multi-kinase inhibitor with potent vascular endothelial growth factor receptor 2 (VEGFR2) inhibition pazopanib is a Food and Drug Administration approved drug with activity in SS. ${ }^{97}$ Combinations of anti-angiogenic agents with immunebased therapies may represent a mechanism to enhance immune responses against CTAs.

\section{FUTURE DIRECTIONS}

As more data demonstrating activity of CTA-targeted vaccines and TCR-based therapies emerges, additional efforts are underway to enhance the efficacy of these therapies and to develop additional ways of targeting CTAs.

CAR-T cells have also been used in various trials as experimental treatments to target CTA positive cancers. A major disadvantage of CAR-T cells is that they are limited to antigens that are expressed on the cell surface. ${ }^{98}$ While this can greatly limit the amount and type of antigens that these cells can target, CAR-T cells have been shown to elicit a greater release of cytokines, such as IL-2. ${ }^{99}$ CAR-T cells targeted against NY-ESO-1 have shown early efficacy in an in vivo murine model of NY-ESO-1 positive multiple myeloma. ${ }^{100}$ This study also included a NY-ESO-1 vaccine that was used to increase the efficacy and persistence of the CAR-T Cells. Given the preliminary success of autologous T-cells targeting NY-ESO-1 in patients with SS, additional study with CAR-T for NY-ESO-1 expressing SS is warranted and may allow a mechanism to overcome the HLA restriction that limits the applicability of autologous T-cell products.

Rational combinations of TCR-targeted therapies with other agents to modulate the immune microenvironment will be one focus of future research. Use of the cytokine IL-2, which promotes activation and cell growth of the transferred $\mathrm{T}$ lymphocytes, in conjunction with $\mathrm{T}$ cell therapy shows promise with positive responses by response evaluation criteria in solid tumors (RECIST) criteria in some patients. ${ }^{66} 68$ The use of checkpoint inhibitors has 
also been proposed for concurrent use with other $\mathrm{T}$ cell or vaccine-based therapies. ${ }^{85}$ SS has low expression of checkpoint markers such as PD-1 and PD-L1, but the use of checkpoint inhibitors might aid in $\mathrm{T}$ cell or vaccine therapy, especially those who are recurrent or are metastatic. ${ }^{85993}$

Separate from vaccines and cell-based therapies, bispecific TCRs may also present a mechanism to effectively target CTAs without the time constraints that inherently come with vaccine or cell-based therapy. Bispecific TCRs are soluble molecules that are engineered to recognize their specific protein target and have an anti-CD3 base. Although there was one study of an anti-NY-ESO-1-anti-CD3 bispecific soluble TCR (NCT03515551), this molecule is no longer in clinical development. ${ }^{101}$

\section{Conclusions}

CTAs are highly expressed in SS. Their limited expression in normal adult tissues presents an opportunity to target these aberrantly expressed proteins using various forms of immune mediated therapies. Indeed, significant progress has been made in developing CTA-targeted therapies for treatment of SS, in particular with HLA-restricted autologous T-cells targeting NY-ESO-1 and MAGE-A4. However, the inherent limitations of these therapies which are HLA restricted and require expression of the targeted CTA leave a large swath of patients without effective therapies. Additionally, intrinsic immune properties of SS and the sarcoma immune microenvironment allow some tumors to evade the immune system. To develop reliable CTAtargeted therapies that will be more broadly applicable and effective, future strategies will need to focus on rationally designed combinations that maximize the immune response against CTAs.

Twitter Seth M Pollack @immunosarc and Michael J Wagner @wagsmd

Acknowledgements Figure 1 was created in Biorender.com.

Contributors All authors contributed to the writing of the original manuscript and reviewed the final version.

Funding The authors have not declared a specific grant for this research from any funding agency in the public, commercial or not-for-profit sectors.

Competing interests SMP receives research funding from Merck, EMD Serono, Incyte, Presage, Janssen, Oncosec and Juno. He has consulting, honoraria and advisory activity with GlaxoSmithKline, Apexigen, Daiichi Sankyo and T-Knife. MJW, advisory board for Deciphera, Adaptimmune, research support to institution from Adaptimmune, GSK, Athenex and Incyte. GM has no conflicts to declare.

Patient consent for publication Not required.

Provenance and peer review Not commissioned; externally peer reviewed.

Open access This is an open access article distributed in accordance with the Creative Commons Attribution Non Commercial (CC BY-NC 4.0) license, which permits others to distribute, remix, adapt, build upon this work noncommercially, and license their derivative works on different terms, provided the original work is properly cited, appropriate credit is given, any changes made indicated, and the use is non-commercial. See http://creativecommons.org/ licenses/by-nc/4.0/.

\section{ORCID iDs}

Seth M Pollack http://orcid.org/0000-0002-2466-0607

Michael J Wagner http://orcid.org/0000-0002-0753-9282

\section{REFERENCES}

1 Deshmukh R, Mankin HJ, Singer S. Synovial sarcoma: the importance of size and location for survival. Clin Orthop Relat Res 2004;419:155-61.

2 Wang S, Song R, Sun T, et al. Survival changes in patients with synovial sarcoma, 1983-2012. J Cancer 2017;8:1759-68.

3 Skytting B. Synovial sarcoma. A Scandinavian sarcoma group project. Acta Orthop Scand Supp/ 2000;291:1-28.

4 Jones SF, Whitman RC. Primary sarcoma of the lower end of the femur involving the synovial membrane: with a critical review of the literature of synovial sarcoma report of one case with complete pathological and radiographic examination. Ann Surg 1914;60:440-50.

5 Barrott JJ, Illum BE, Jin H, et al. Paracrine osteoprotegerin and $\beta$-catenin stabilization support synovial sarcomagenesis in periosteal cells. J Clin Invest 2018;128:207-18.

6 Nielsen OS, Judson I, van Hoesel Q, et al. Effect of high-dose ifosfamide in advanced soft tissue sarcomas. A multicentre phase II study of the EORTC soft tissue and bone sarcoma group. Eur $J$ Cancer 2000;36:61-7.

7 van der Graaf WTA, Blay J-Y, Chawla SP, et al. Pazopanib for metastatic soft-tissue sarcoma (PALETTE): a randomised, doubleblind, placebo-controlled phase 3 trial. Lancet 2012;379:1879-86.

8 Kawai A, Araki N, Sugiura H, et al. Trabectedin monotherapy after standard chemotherapy versus best supportive care in patients with advanced, translocation-related sarcoma: a randomised, openlabel, phase 2 study. Lancet Oncol 2015;16:406-16.

9 Pollack SM, Somaiah N, Araujo DM, et al. Clinical outcomes of patients with advanced synovial sarcoma or myxoid/round cell liposarcoma treated at major cancer centers in the United States. Cancer Med 2020;9:4593-602.

10 Tamaki S, Fukuta M, Sekiguchi K, et al. Ss18-Ssx, the oncogenic fusion protein in synovial sarcoma, is a cellular context-dependent epigenetic modifier. PLoS One 2015;10:e0142991.

11 Middeljans E, Wan X, Jansen PW, et al. Ss18 together with animalspecific factors defines human BAF-type SWI/SNF complexes. PLoS One 2012;7:e33834.

12 Su L, Sampaio AV, Jones KB, et al. Deconstruction of the SS18SSX fusion oncoprotein complex: insights into disease etiology and therapeutics. Cancer Cell 2012;21:333-47.

13 Nacev BA, Jones KB, Intlekofer AM. Nielsen to: the epigenomics of sarcoma. Nat Rev Cancer 2020;20:608-23.

14 McBride MJ, Pulice JL, Beird HC, et al. The SS18-SSX fusion oncoprotein hijacks BAF complex targeting and function to drive synovial sarcoma. Cancer Cell 2018;33:1128-41.

15 Kadoch C, Crabtree GR. Reversible disruption of mSWI/SNF (BAF) complexes by the SS18-SSX oncogenic fusion in synovial sarcoma. Cell 2013;153:71-85.

16 Uyttenhove C, Maryanski J, Boon T. Escape of mouse mastocytoma P815 after nearly complete rejection is due to antigen-loss variants rather than immunosuppression. J Exp Med 1983;157:1040-52.

17 van der Bruggen P, Traversari C, Chomez P, et al. A gene encoding an antigen recognized by cytolytic $T$ lymphocytes on a human melanoma. Science 1991;254:1643-7.

18 Chen Y-T, Scanlan MJ, Sahin U, et al. A testicular antigen aberrantly expressed in human cancers detected by autologous antibody screening. Proc Natl Acad Sci U S A 1997;94:1914-8.

19 Chen YT, Güre AO, Tsang S, et al. Identification of multiple cancer/ testis antigens by allogeneic antibody screening of a melanoma cell line library. Proc Natl Acad Sci U S A 1998;95:6919-23.

20 Almeida LG, Sakabe NJ, deOliveira AR, et al. CTdatabase: a knowledge-base of high-throughput and curated data on cancertestis antigens. Nucleic Acids Res 2009;37:D816-9.

21 Tammela J, Jungbluth AA, Qian F. SCP-1 cancer/testis antigen is a prognostic indicator and a candidate target for immunotherapy in epithelial ovarian cancer. Cancer Immun 2004;4:10.

22 Ollinger R, Alsheimer M, Benavente R. Mammalian protein SCP1 forms synaptonemal complex-like structures in the absence of meiotic chromosomes. Mol Biol Cell 2005;16:212-7.

23 Gjerstorff MF, Kock K, Nielsen O, et al. Mage-A1, GAGE and NYESO-1 cancer/testis antigen expression during human gonadal development. Human Reproduction 2007;22:953-60.

24 Barker PA, Salehi A. The MAGE proteins: emerging roles in cell cycle progression, apoptosis, and neurogenetic disease. J Neurosci Res 2002;67:705-12.

25 De Smet C, De Backer O, Faraoni I, et al. The activation of human gene MAGE-1 in tumor cells is correlated with genome-wide demethylation. Proc Natl Acad Sci U S A 1996;93:7149-53.

26 Simpson AJG, Caballero OL, Jungbluth A, et al. Cancer/ Testis antigens, gametogenesis and cancer. Nat Rev Cancer 2005;5:615-25. 
27 Jungbluth AA, Chen YT, Stockert E, et al. Immunohistochemical analysis of NY-ESO-1 antigen expression in normal and malignant human tissues. Int J Cancer 2001;92:856-60.

28 Vaughan HA, Svobodova S, Macgregor D, et al. Immunohistochemical and molecular analysis of human melanomas for expression of the human cancer-testis antigens NY-ESO-1 and LAGE-1. Clin Cancer Res 2004;10:8396-404.

29 Grigoriadis A, Caballero OL, Hoek KS, et al. CT-X antigen expression in human breast cancer. Proc Natl Acad Sci U S A 2009;106:13493-8.

30 Yamada A, Kataoka A, Shichijo S, et al. Expression ofMAGE-1, MAGE-2, MAGE-3/-6 andMAGE-4A/-4B genes in ovarian tumors. Int J Cancer 1995;64:388-93.

31 Kurashige T, Noguchi Y, Saika T, et al. Ny-Eso-1 expression and immunogenicity associated with transitional cell carcinoma: correlation with tumor grade. Cancer Res 2001;61:4671-4.

32 Melloni G, Ferreri AJM, Russo V, et al. Prognostic significance of cancer-testis gene expression in resected non-small cell lung cancer patients. Oncol Rep 2004;12:145-51.

33 Gure AO, Chua R, Williamson B, et al. Cancer-Testis genes are coordinately expressed and are markers of poor outcome in nonsmall cell lung cancer. Clin Cancer Res 2005;11:8055-62.

34 Taylor BJ, Reiman T, Pittman JA. Pilarski LM: SSX cancer testis antigens are expressed in most multiple myeloma patients: co-expression of SSX1, 2, 4, and 5 correlates with adverse prognosis and high frequencies of SSX-positive PCs. J Immunother 2005;28:564-75.

35 Chen $\mathrm{CH}$, Huang GT, Lee HS, et al. High frequency of expression of MAGE genes in human hepatocellular carcinoma. Liver 1999;19:110-4

36 Jäger E, Gnjatic S, Nagata Y, et al. Induction of primary NY-ESO-1 immunity: CD8+ T lymphocyte and antibody responses in peptidevaccinated patients with NY-ESO-1+ cancers. Proc Natl Acad Sci U S A 2000;97:12198-203.

37 Pollack SM, Jungbluth AA, Hoch BL, et al. Ny-Eso-1 is a ubiquitous immunotherapeutic target antigen for patients with myxoid/round cell liposarcoma. Cancer 2012;118:4564-70.

38 Maxfield KE, Taus PJ, Corcoran K, et al. Comprehensive functional characterization of cancer-testis antigens defines obligate participation in multiple hallmarks of cancer. Nat Commun 2015;6:8840.

39 Cho HJ, Caballero OL, Gnjatic S, et al. Physical interaction of two cancer-testis antigens, MAGE-C1 (CT7) and NY-ESO-1 (CT6). Cancer Immun 2006;6:12.

40 Doyle JM, Gao J, Wang J, et al. MAGE-RING protein complexes comprise a family of E3 ubiquitin ligases. Mol Cell 2010;39:963-74.

41 lura K, Maekawa A, Kohashi K, et al. Cancer-Testis antigen expression in synovial sarcoma: NY-ESO-1, PRAME, MAGEA4, and MAGEA1. Hum Pathol 2017:61:130-9.

42 Kakimoto T, Matsumine A, Kageyama S. Sudo A immunohistochemical expression and clinicopathological assessment of the cancer testis antigens NY-ESO-1 and MAGE-A4 in high-grade soft-tissue sarcoma. Oncol Lett 2019;17:3937-43.

43 Jungbluth AA, Antonescu CR, Busam KJ, et al. Monophasic and biphasic synovial sarcomas abundantly express cancer/ testis antigen NY-ESO-1 but not MAGE-A1 or ct7. Int J Cancer 2001;94:252-6.

44 Endo M, de Graaff MA, Ingram DR, et al. Ny-Eso-1 (CTAG1B) expression in mesenchymal tumors. Mod Pathol 2015;28:587-95.

45 Lai J-P, Robbins PF, Raffeld M, et al. Ny-Eso-1 expression in synovial sarcoma and other mesenchymal tumors: significance for NY-ESO-1-based targeted therapy and differential diagnosis. Mod Pathol 2012;25:854-8.

46 Sellner L, Chudasama P, Renner M. Screening for the cancer immunotherapy target NY-ESO-1 in soft-tissue sarcoma. Oncology Research and Treatment 2017;40:111.

47 Segal NH, Blachere NE, Guevara-Patiño JA. Houghton an: identification of cancer-testis genes expressed by melanoma and soft tissue sarcoma using bioinformatics. Cancer Immun 2005;5:2.

48 Marcar L, MacLaine NJ, Hupp TR, et al. Mage-A cancer/testis antigens inhibit p53 function by blocking its interaction with chromatin. Cancer Res 2010;70:10362-70.

49 Marcar L, Ihrig B, Hourihan J, et al. MAGE-A cancer/testis antigens inhibit MDM2 ubiquitylation function and promote increased levels of Mdm4. PLoS One 2015;10:e0127713.

50 Jungbluth $A$, Frosina D, Fayad $M$, et al. Cancer testis antigen PRAME is abundantly expressed in metastatic melanoma and other malignancies. Laboratory Investigation 2018;98:702.

51 Luk SJ, Steen DMvander, Hagedoorn RS, et al. Prame and HLA class I expression patterns make synovial sarcoma a suitable target for PRAME specific T-cell receptor gene therapy. Oncoimmunology 2018;7:e1507600

52 Roszik J, Wang W-L, Ravi V, et al. Expression and clinical correlations of PRAME in sarcoma subtypes. Journal of Clinical Oncology 2016;34:11067.

53 Epping MT, Wang L, Edel MJ, et al. The human tumor antigen PRAME is a dominant repressor of retinoic acid receptor signaling. Cell 2005;122:835-47.

54 Ikeda $\mathrm{H}$, Lethé $\mathrm{B}$, Lehmann $\mathrm{F}$, et al. Characterization of an antigen that is recognized on a melanoma showing partial HLA loss by CTL expressing an NK inhibitory receptor. Immunity 1997;6:199-208.

55 D'Arcy P, Maruwge W, Wolahan B, et al. Oncogenic functions of the cancer-testis antigen SSX on the proliferation, survival, and signaling pathways of cancer cells. PLoS One 2014;9:e95136.

56 Kawai A, Woodruff J, Healey JH, et al. SYT-SSX Gene Fusion as a Determinant of Morphology and Prognosis in Synovial Sarcoma. $N$ Engl J Med Overseas Ed 1998;338:153-60.

57 Somaiah N, Block MS, Kim JW, et al. First-In-class, first-in-human study evaluating LV305, a dendritic-cell tropic lentiviral vector, in sarcoma and other solid tumors expressing NY-ESO-1. Clin Cancer Res 2019;25:5808-17.

58 Chawla S, Van Tine BA, Pollack S. A phase 2 study of CMB305 and atezolizumab in NY-ESO-1+ soft tissue sarcoma: interim analysis of immunogenicity, tumor control and survival. Ann Oncol 2017;28:v523.

59 Somaiah N, Chawla SP, Block MS, et al. A phase 1B study evaluating the safety, tolerability, and immunogenicity of CMB305, a Lentiviral-Based prime-boost vaccine regimen, in patients with locally advanced, relapsed, or metastatic cancer expressing NYESO-1. Oncoimmunology 2020;9:1847846.

60 Chawla SP, Van Tine BA, Pollack S, et al. A phase II randomized study of CMB305 and atezolizumab versus atezolizumab in NYESO-1 ${ }^{+}$soft tissue sarcoma: analysis of immunogenicity, tumor control, and patient survival. J Clin Oncol 2019;37:11011.

61 Ishihara M, Tono Y, Miyahara Y, et al. First-In-Human phase I clinical trial of the NY-ESO-1 protein cancer vaccine with NOD2 and TLR9 stimulants in patients with NY-ESO-1-expressing refractory solid tumors. Cancer Immunol Immunother 2020;69:663-75.

62 Kawaguchi S, Tsukahara T, Ida K, et al. Syt-Ssx breakpoint peptide vaccines in patients with synovial sarcoma: a study from the Japanese musculoskeletal Oncology Group. Cancer Sci 2012;103:1625-30.

63 Ebert LM, MacRaild SE, Zanker D, et al. A cancer vaccine induces expansion of NY-ESO-1-specific regulatory T cells in patients with advanced melanoma. PLoS One 2012;7:e48424.

64 Pavlick A, Blazquez AB, Meseck M, et al. Combined vaccination with NY-ESO-1 protein, Poly-ICLC, and Montanide improves humoral and cellular immune responses in patients with high-risk melanoma. Cancer Immunol Res 2020;8:70-80.

65 Pollack SM, Jones RL, Farrar EA, et al. Tetramer guided, cell sorter assisted production of clinical grade autologous NY-ESO-1 specific CD8(+) T cells. J Immunother Cancer 2014;2:36.

66 Robbins PF, Morgan RA, Feldman SA, et al. Tumor regression in patients with metastatic synovial cell sarcoma and melanoma using genetically engineered lymphocytes reactive with NY-ESO-1. JCO 2011;29:917-24.

67 Robbins PF, Li YF, El-Gamil M, et al. Single and dual amino acid substitutions in TCR CDRs can enhance antigen-specific T cell functions. J Immunol 2008;180:6116-31.

68 Robbins PF, Kassim SH, Tran TLN, et al. A pilot trial using lymphocytes genetically engineered with an NY-ESO-1-reactive Tcell receptor: long-term follow-up and correlates with response. Clin Cancer Res 2015;21:1019-27.

69 D'Angelo SP, Melchiori L, Merchant MS, et al. Antitumor activity associated with prolonged persistence of adoptively transferred

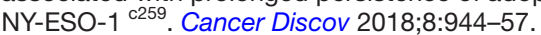

70 Joseph J, Nathenson MJ, Trinh VA, et al. Guillain-Barre syndrome observed with adoptive transfer of lymphocytes genetically engineered with an NY-ESO-1 reactive T-cell receptor. J Immunother Cancer 2019:7:296.

71 Butler MO, Sotov V, Saibil S. Adoptive T cell therapy with TBI-1301 results in gene-engineered $\mathrm{T}$ cell persistence and anti-tumour responses in patients with NY-ESO-1 expressing solid tumours. Ann Oncol 2019;30:v481.

72 Hattori H, Ishihara M, Kitano S, et al. A novel affinity-enhanced NYESO-1-targeting TCR-redirected T cell transfer exhibited early-onset cytokine release syndrome and subsequent tumour responses in synovial sarcoma patients. Ann Oncol 2019;30:v481.

73 Sanderson JP, Crowley DJ, Wiedermann GE, et al. Preclinical evaluation of an affinity-enhanced MAGE-A4-specific T-cell receptor for adoptive T-cell therapy. Oncoimmunology 2020;9:1682381. 
74 Hong DS, Van Tine BA, Olszanski AJ, et al. Phase I dose escalation and expansion trial to assess the safety and efficacy of ADP-A2M4 spear T cells in advanced solid tumors. JCO 2020;38:102.

75 Van Tine BA, Hong DS. Durable responses in patients with synovial sarcoma in the phase I trial of ADP-A2M4 (MAGE-A4) in: connective tissue oncology Society. virtual meeting 2020.

76 Morgan RA, Chinnasamy N, Abate-Daga D, et al. Cancer regression and neurological toxicity following anti-MAGE-A3 TCR gene therapy. J Immunother 2013;36:133-51.

77 Stadtmauer EA, Fraietta JA, Davis MM, et al. CRISPR-engineered T cells in patients with refractory cancer. Science2020;367:eaba7365.

78 Vakulskas CA, Dever DP, Rettig GR, et al. A high-fidelity Cas9 mutant delivered as a ribonucleoprotein complex enables efficient gene editing in human hematopoietic stem and progenitor cells. Nat Med 2018;24:1216-24.

79 Cao K, Hollenbach J, Shi X, et al. Analysis of the frequencies of HLA-A, B, and $C$ alleles and haplotypes in the five major ethnic groups of the United States reveals high levels of diversity in these loci and contrasting distribution patterns in these populations. Hum Immunol 2001;62:1009-30.

80 Dudley ME, Yang JC, Sherry R, et al. Adoptive cell therapy for patients with metastatic melanoma: evaluation of intensive myeloablative chemoradiation preparative regimens. JCO 2008;26:5233-9.

81 Ramachandran I, Lowther DE, Dryer-Minnerly R, et al. Systemic and local immunity following adoptive transfer of NY-ESO-1 spear T cells in synovial sarcoma. J Immunother Cancer 2019;7:276.

82 Binnewies M, Roberts EW, Kersten K, et al. Understanding the tumor immune microenvironment (time) for effective therapy. Nat Med 2018;24:541-50.

83 He M, Abro B, Kaushal M, et al. Tumor mutation burden and checkpoint immunotherapy markers in primary and metastatic synovial sarcoma. Hum Pathol 2020;100:15-23.

84 Pollack SM, He Q, Yearley JH, et al. T-Cell infiltration and clonality correlate with programmed cell death protein 1 and programmed death-ligand 1 expression in patients with soft tissue sarcomas. Cancer 2017;123:3291-304.

85 D'Angelo SP, Shoushtari AN, Agaram NP, et al. Prevalence of tumorinfiltrating lymphocytes and PD-L1 expression in the soft tissue sarcoma microenvironment. Hum Pathol 2015;46:357-65.

86 Oike N, Kawashima H, Ogose A, et al. Prognostic impact of the tumor immune microenvironment in synovial sarcoma. Cancer $\mathrm{Sci}$ 2018;109:3043-54.

87 Wedekind MF, Haworth KB, Arnold M, et al. Immune profiles of desmoplastic small round cell tumor and synovial sarcoma suggest different immunotherapeutic susceptibility upfront compared to relapse specimens. Pediatr Blood Cancer 2018;65:e27313

88 van Erp AEM, Versleijen-Jonkers YMH, Hillebrandt-Roeffen MHS. Expression and clinical association of programmed cell death-1, programmed death-ligand-1 and CD8. Oncotarget 2017;8:71371-84.

89 Nowicki TS, Akiyama R, Huang RR, et al. Infiltration of CD8 T cells and expression of PD-1 and PD-L1 in synovial sarcoma. Cancer Immunol Res 2017;5:118-26.
90 Zhang S, Cooper S, Donahue B, et al. Interferon gamma induced transformation of the cold tumor microenvironment in patients with NY-ESO-1 expressing sarcomas. Cancer Res 2018;78.

91 Zhang S, Kohli K, Black RG, et al. Systemic interferon- $\gamma$ increases $\mathrm{MHC}$ class I expression and T-cell infiltration in cold tumors: results of a phase 0 clinical trial. Cancer Immunol Res 2019;7:1237-43.

92 Schroeder BA, Black RG, Spadinger S, et al. Histiocyte predominant myocarditis resulting from the addition of interferon gamma to cyclophosphamide-based lymphodepletion for adoptive cellular therapy. J Immunother Cancer 2020;8:e000247.

93 Maki RG, Jungbluth AA, Gnjatic S, et al. A pilot study of anti-CTLA4 antibody ipilimumab in patients with synovial sarcoma. Sarcoma 2013;2013:168145

94 Tawbi HA, Burgess M, Bolejack V, et al. Pembrolizumab in advanced soft-tissue sarcoma and bone sarcoma (SARC028): a multicentre, two-cohort, single-arm, open-label, phase 2 trial. Lancet Oncol 2017;18:1493-501.

95 Barrott JJ, Kafchinski LA, Jin H, et al. Modeling synovial sarcoma metastasis in the mouse: PI3'-lipid signaling and inflammation. $J$ Exp Med 2016;213:2989-3005.

96 Lee WS, Yang $\mathrm{H}$, Chon HJ, et al. Combination of anti-angiogenic therapy and immune checkpoint blockade normalizes vascularimmune crosstalk to potentiate cancer immunity. Exp Mol Med 2020;52:1475-85.

97 Sroussi M, De Percin S, Grecea AM, et al. Pazopanib in advanced or metastatic synovial sarcoma: the Gustave Roussy experience. Annals of Oncology 2018;29:viii589-90.

98 Harris DT, Kranz DM. Adoptive T cell therapies: a comparison of T cell receptors and chimeric antigen receptors. Trends Pharmacol Sci 2016;37:220-30.

99 Harris DT, Hager MV, Smith SN, et al. Comparison of T cell activities mediated by human TCRs and cars that use the same recognition domains. J.i. 2018;200:1088-100.

100 Patel K, Olivares S, Singh H, et al. Combination immunotherapy with NY-ESO-1-specific CAR + T cells with T-cell vaccine improves anti-myeloma effect. Blood 2016;128:3366.

101 Lopez J, Shinde R, Burgess MA. Trial in progress: first-in-human study of a novel anti-NY-ESO-1-anti-CD3, TCR-based bispecific (IMCnyeso) as monotherapy in NY-ESO-1/LAGE-1A-positive advanced solid tumours (IMCnyeso-101). Ann Oncol 2019;30:v532.

102 Ayyoub M, Taub RN, Keohan M-L. The frequent expression of cancer/testis antigens provides opportunities for immunotherapeutic targeting of sarcoma. Cancer Immun 2004;4:7.

103 Antonescu CR, Busam KJ, Iversen K, et al. Mage antigen expression in monophasic and biphasic synovial sarcoma. Hum Pathol 2002;33:225-9.

104 Guillou L, Coindre J, Gallagher G, et al. Detection of the synovial sarcoma translocation $\mathrm{t}(\mathrm{X} ; 18)$ (SYT;SSX) in paraffin-embedded tissues using reverse transcriptase-polymerase chain reaction: a reliable and powerful diagnostic tool for pathologists. A molecular analysis of 221 mesenchymal tumors fixed in different fixatives. Hum Pathol 2001;32:105-12.

105 Wei Y, Wang J, Zhu X, et al. Detection of SYT-SSX fusion transcripts in paraffin-embedded tissues of synovial sarcoma by reverse transcription-polymerase chain reaction. Chin Med $J$ 2002:115:1043-7. 